\title{
Head tracked retroreflecting 3D display
}

\author{
Phil Surman (SID Member) \\ Sally Day (SID Senior Member) \\ Xianzi Liu \\ Joshua Benjamin (SID Student Member) \\ Hakan Urey (SID Senior Member) \\ Kaan Aksit (SID Member)
}

\begin{abstract}
In this paper, we describe a single-user glasses-free (autostereoscopic) 3D display where images from a pair of picoprojectors are projected on to a retroreflecting screen. Real images of the projector lenses formed at the viewer's eyes produce exit pupils that follow the eye positions by the projectors moving laterally under the control of a head tracker. This provides the viewer with a comfortable degree of head movement. The retroreflecting screen, display hardware, infrared head tracker, and means of stabilizing the image position on the screen are explained. The performance of the display in terms of crosstalk, resolution, image distortion, and other parameters is described. Finally, applications of this display type are suggested.
\end{abstract}

Keywords - autostereoscopic, retroreflector, picoprojector, exit pupils, head tracking, infrared.

DOI \# 10.1002/jsid.295

\section{Background}

An autostereoscopic display can be produced by various methods. Holography has the potential to produce a perfect reproduction of the original scene by wavefront reconstruction; however, it is complex and requires the display of large amounts of information. A light field display can reproduce the original scene in such a way that differences are indistinguishable to the human visual system. In these displays, the light emitted from a point on the screen varies with direction. Ideally, parallax would be produced in two directions so that as an observer moves in both the horizontal and vertical directions, the image appears to vary, thus providing motion parallax, the ability to "look around" an object. This requires the display of a large amount of information, and vertical parallax is dispensed within many displays on the assumption that the viewers' heads are oriented in the usual direction for a standing or sitting position and the display is in its usual orientation, for instance as for a television set or cinema. ${ }^{1,2}$ For handheld applications, it can be preferable to have parallax in all directions, and some displays address this. ${ }^{3}$

A light field display shows continuous motion parallax so that as the viewer's eye moves in relation to the display, the change of the appearance of the image appears to be smooth. This has the benefit that the eye focuses at the same distance at which it converges so that accommodation/convergence conflict that can produce eyestrain in stereoscopic displays will not occur. ${ }^{4}$

Another class of display is multiview where motion parallax can be produced by displaying a series of discrete images across the viewing field. ${ }^{5,6}$ These displays represent the current state of the art and require the display of less information than a light field display but have the disadvantage of limited depth of field so that when an object away from the plane of the screen is displayed, multiple images are perceived because of crosstalk. Multiview displays can provide useful viewing areas over a fairly large proportion of the viewing field in front of the screen as the series of viewing zones is repeated ${ }^{7}$; however, the complete viewing field cannot be covered.

In order to overcome various issues, for example, large amounts of information having to be displayed, limited viewing field area, and limited depth of field, the display can show a single image pair where this can be observed at certain locations, referred to here as exit pupils. As the user moves, the exit pupils can follow their eye positions under the control of a head tracker The use of a single image pair has the possible disadvantage of not providing inherent motion parallax, although this could be obtained for one user by rendering the image information in accordance with the viewer's position. This type of display also suffers from accommodation/ convergence conflict as the user's eyes focus on the screen but converge at the apparent distance of a displayed object. The effect of this conflict however can be minimized by keeping the disparity between the two images within certain limits. ${ }^{8-10}$

As it is not comfortable for users to remain in one position, it is preferable to move the exit pupils to follow the positions of their eyes. This can be achieved by determining the eye positions and moving the projectors so that the exit pupils always occupy the same positions as the eyes. This makes the display a head-tracked type; these have been under development in different forms for many years with the earliest reference found to date being that of Schwartz in $1985 .^{11}$ There have

Received 01/1/2014; accepted 03/27/2015

P. Surman is with Nanyang Technological University; e-mail: psurman@ntu.edu.sg.

S. Day, X. Liu and J. Benjamin are with University College London, Department of Electronic and Electrical Engineering, London, UK

H. Urey and K. Aksit are with Koç University, Department of Electrical Engineering, Istanbul, Turkey

(c) Copyright 2015 Society for Information Display 1071-0922/15/0000\$1.00. 
been many others since then including those of Sharp ${ }^{12}$ and Massachusetts Institute of Technology (MIT). ${ }^{13}$ Microsoft has developed a head-tracked 3D display ${ }^{14}$ based on the Wedge waveguide technology originally developed at Cambridge University. LG produce a head-tracked 3D monitor ${ }^{15}$ and a Toshiba head-tracked laptop. ${ }^{16}$

Retroreflecting screen displays where the projectors are mounted to the users' heads have been developed. ${ }^{1718}$ Two papers are suggested for the references. Further work was carried out in J. Rolland's group, ${ }^{19}$ and they provide a review of implementations and applications that developed the group, and Martins et al. ${ }^{20}$ extend the concepts to a fully mobile display. An autostereoscopic display system using pupil imaging with a curved mirror and a $1920 \times 1200$ liquid crystal on silicon spatial light modulators has been described. ${ }^{21}$

\section{Principle of operation}

The availability of small and relatively inexpensive picoprojectors has encouraged the authors to develop a 3D display based on these. The principle of using projectors in conjunction with a retroreflecting screen where light is reflected back at the same angle it enters was described by Okoshi in $1976 .^{22}$ A pair of projectors is used to form exit pupils in the vicinity of the projector lens so that eyes located in these regions see either a left or a right image over the complete area of the screen.

With only a retroreflector as the screen, light would return to the vicinity of the projector lenses, and the viewers' eyes could not be located in these regions. In order for the viewer's eyes to be situated within an exit pupil region, and also to enable vertical head movement, the light is diffused vertically at the screen as shown in Fig. 1. By itself, this configuration would be difficult to implement as the projectors would be located at the same distance from the screen as the viewer's eyes. The use of mirrors as shown in the figure overcomes this problem.

In order to provide a comfortable degree of head movement, the exit pupils can be made to follow the viewer's eye positions in the $\mathrm{X}$ direction by moving the projector laterally on a stage. Although there is no tracking in the $\mathrm{Z}$ direction, the viewer has a reasonable degree of movement due to the elongated diamond-shaped section exit pupils.

The relatively simple single viewer display described here has been developed using same tracker technology as the multi-user HELIUM3D display.

Vertically elongated real images of the picoprojector lenses form the exit pupils shown projected on to the head of a viewer in Fig. 2. The lenses of the projectors are only $8 \mathrm{~mm}$ diameter, and if the retroreflectors had an ideal characteristic, then narrow exit pupils would be formed with viewers having very little lateral freedom of movement.

\section{Retroreflector screen}

Retroreflectors generally operate using either the corner cube principle $\mathrm{e}^{23-25}$ or a spherical refracting surface with a spherical reflecting surface located behind it ${ }^{26}$; familiar examples of these are the reflectors found on bicycles and "cats eyes" in the road. A corner cube is an arrangement of three orthogonal mirrors that form the corner of a cube. Light is reflected by the mirrors, generally three times, so that it is reflected back at the same angle at which it entered the mirrors.

This is the type of reflector used in the prototype as it has been found to have the superior reflection characteristics necessary for this application. The reflecting surfaces are the rear surface of a refracting medium screen where the front surface is flat and the light is prevented from exiting the rear surface by total internal reflection thus making the reflecting regions effectively prisms.

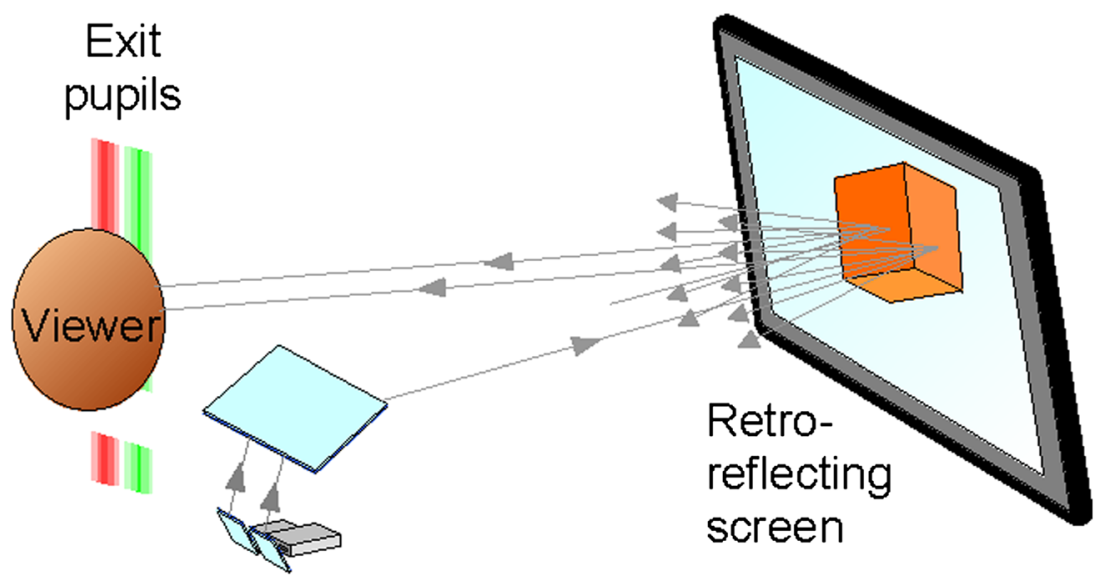

FIGURE 1 - Retroreflector display. An image pupil pair is created by forming real images of the projection lenses with a retroreflector and expanding these vertically with a lenticular screen. 


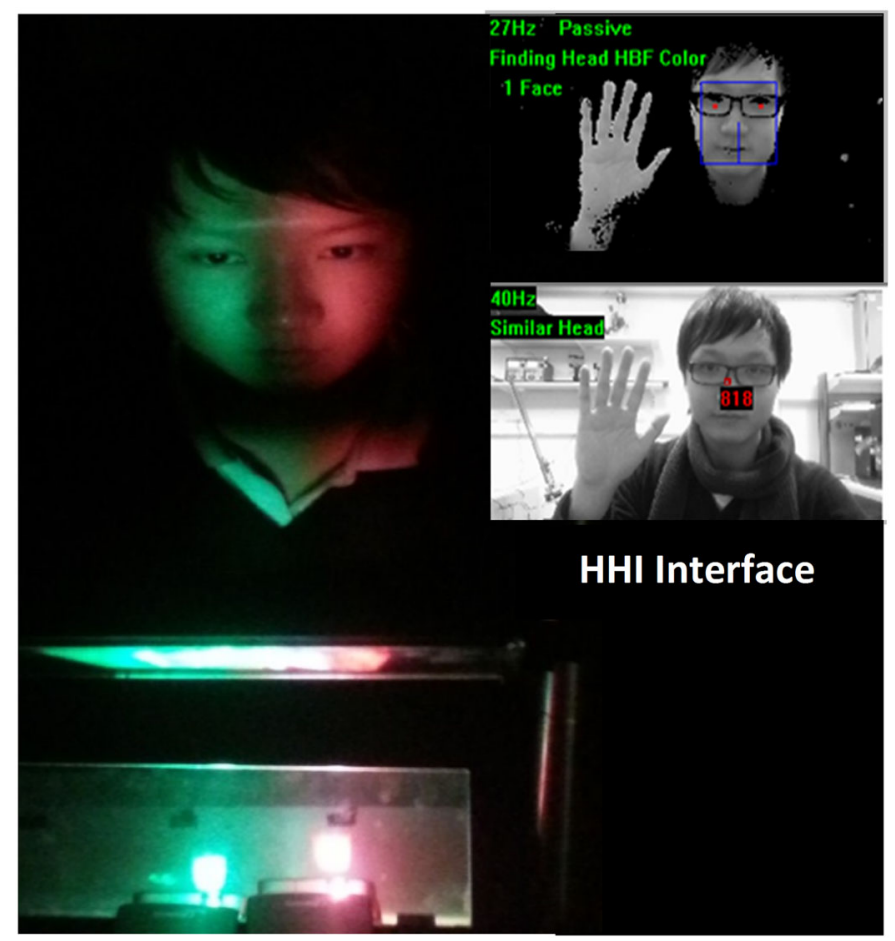

FIGURE 2 - Head tracking. Left: exit pupil pair on viewer's head. Right: section of Fraunhofer $\mathrm{HHI}$ single camera tracking interface.

Figure 3 shows micrographs of the material used for the screen which is white sheeting manufactured by $3 \mathrm{M}$ for use in traffic signs. Figure 3(a) shows the prisms where the dark tips of the prisms are out of focus, with the boundaries between them in focus.

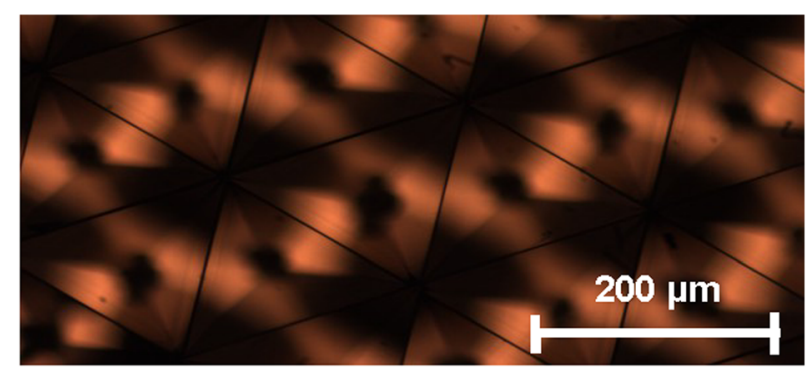

(a) Prisms

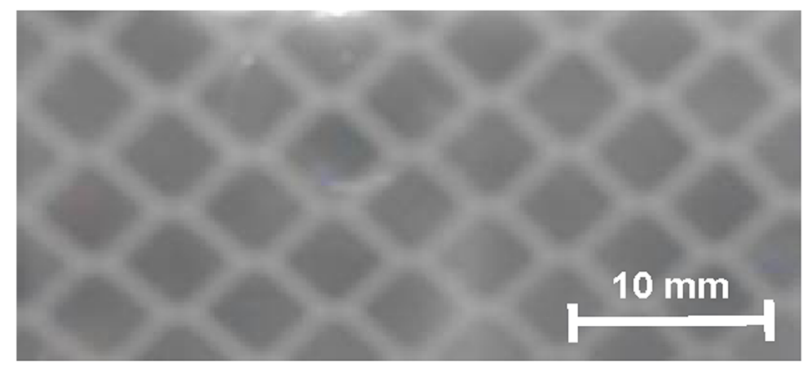

(b) Larger-scale Structure

FIGURE 3 - Retroreflector structure. (a) The pyramid-shaped prisms have base side lengths of $200 \mu \mathrm{m}$ and are arranged in hexagonal configurations. (b) Larger scale visible structure.
In order to determine the suitability of a particular retroreflector material for use in this type of display, it must be characterized. The most important characteristic is the amount of spreading of the reflected beam at the viewing distance of $1.5 \mathrm{~m}$; if this is excessive, then the produced crosstalk will be unacceptable. Crosstalk is where the left image is seen as a dim ghost image by the right eye and vice versa. ${ }^{27}$ If the level of crosstalk is too high, it is difficult for the brain to fuse the two images, and also, viewer discomfort can occur. The acceptable level of crosstalk is dependent on image brightness and content but a typical value is around $5 \% .{ }^{28}$ The requirement for the retroreflector is that the reflected intensity is less than $5 \%$ of its maximum value at a position that is the eye spacing distance from the axis and at $1.5 \mathrm{~m}$ from the screen.

In this particular application, it is useful to have some spread as this allows more freedom of lateral head movement and also requires lower tracker precision. Figure 4(a) shows the spread of a laser beam at $1 \mathrm{~m}$ from the retroreflector screen. It is also useful to know the performance of the retroreflector off-axis as the expanding ray bundle from the projector only lands at normal incidence to the screen at its center. Figure $4(\mathrm{~b})$ is the plot of the variation in reflectance with angle of incidence.

The close-up of the screen is shown in Fig. 5(a) and its performance in Fig. 5(b). The exit pupils are extended vertically with a lenticular screen having horizontally aligned lenses located in front of the retroreflecting screen as in Fig. 5(a). Light directions are maintained in the horizontal direction but are spread vertically. In Fig. 5(b), it can be seen that the input beam is located in the center of the output beam in the vertical direction and both are at angle $\theta$ to the screen normal.

The screen brightness is greatly enhanced with the use of a retroreflecting screen as the light from each projector is concentrated into a vertical zone approximately $60 \mathrm{~mm}$ wide, as opposed to a complete hemisphere of $2 \pi$ steradians. Very high screen gains of between 1000 and 10,000 have been reported for retroreflecting screens ${ }^{20}$ but the maximum theoretical value of the authors' display is likely to be considerably less than 1000. Nevertheless, the observed brightness from the projectors, which would normally be providing luminance in the region of $20 \mathrm{~cd} . \mathrm{m}^{-2}$ if a conventional screen is used, is readily viewed at a screen distance of $1.5 \mathrm{~m}$ and under bright indoor ambient lighting conditions.

There are various factors that have a bearing on the display performance: the principal being, the appearance of fringing on the screen, head tracking to enable viewer movement, specular reflection from the screen, and compensation for lateral image shift due to the projectors' movement.

Moiré fringing is due to interference between the pattern of the mirrors on the retroreflector and the diffuser lenses. The effects of this are difficult to predict by modeling, and the most effective technique is to increase the separation between the retroreflecting screen and the diffuser until the fringing becomes imperceptible. 


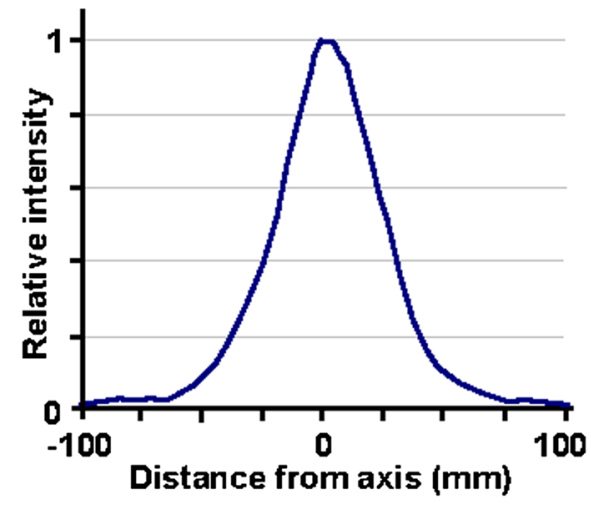

(a) Spread at $1 \mathrm{~m}$

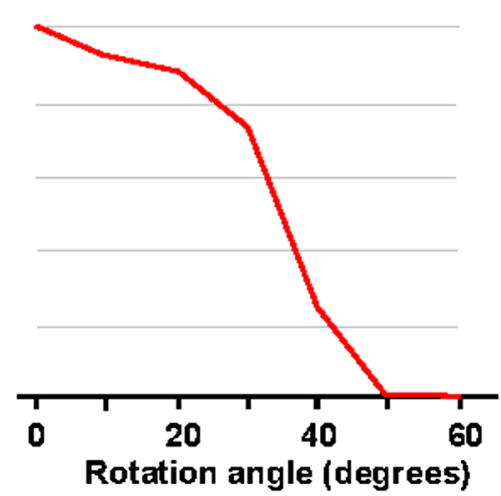

(b) Reflectance vs Angle of Incidence

FIGURE 4 - Retroreflector characteristics. (a) Spread at $1 \mathrm{~m}$ from a laser beam normal to the retroreflector. (b) Decrease in reflectivity as retroreflector is rotated.

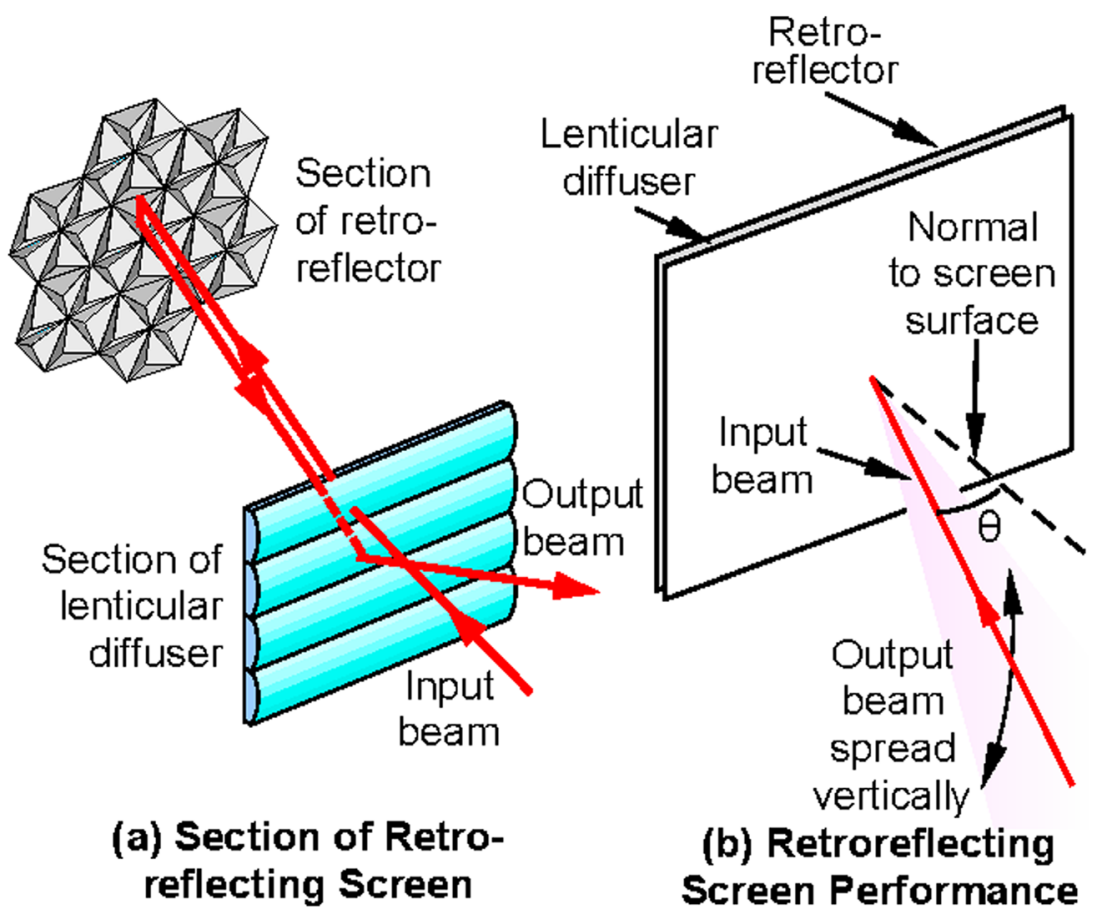

FIGURE 5 - Retroreflecting screen assembly. (a) Screen comprises corner cube array and vertical diffuser. (b) Angle $\theta$ is preserved by the exit beam but the rays are diffused vertically.

If the screen is oriented vertically, bright vertical lines are observed because of specular reflection from the screen components (Fig. 6(a)), in particular from the front surface of the lenticular screen. The visibility of the reflection can be eliminated by tilting the top of the screen forward as shown in Fig. 6(b). When the screen is tilted around $20^{\circ}$ to the vertical, the lines become imperceptible. This gives a slight intensity reduction of around $10 \%$. At this angle, the image on the screen is subject to considerable keystone distortion. However, this is not noticeable as the viewer's eyes are located close to the virtual position of the projector as shown in Fig. 6 (b) so that the distortion of the image is compensated for; the bottom of the screen where the image is actually wider than it is at the top subtends around the same angle as the top because of it being further away from the viewer.

\section{Display hardware}

Lateral viewer head movement is enabled by mounting the projectors on to a stage that can move laterally; the mechanism is shown in Fig. 7. The maximum speed requirement is assumed to be $200 \mathrm{~mm} \mathrm{~s}^{-1}$ as it is unlikely that a viewer would move their head faster than this under normal conditions. The overall positional accuracy required is $\pm 10 \mathrm{~mm}$ as 


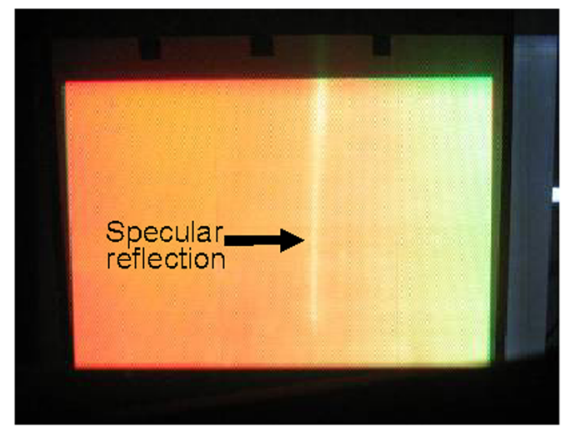

(a) Appearance on Screen

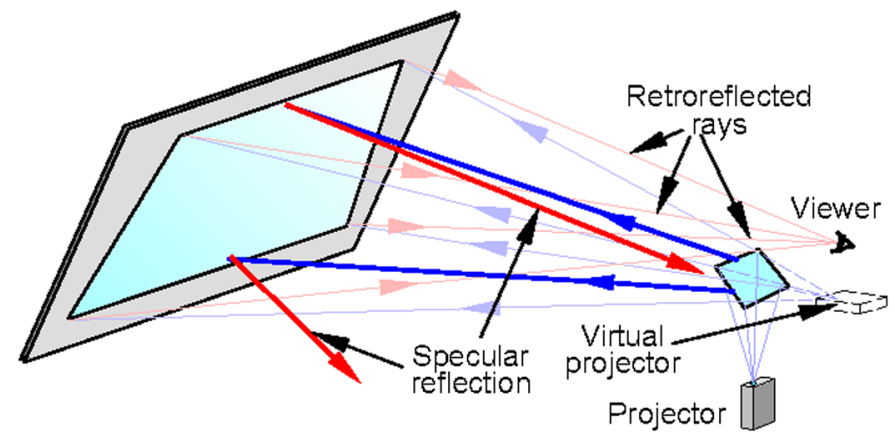

(b) Elimination of Reflected Rays

FIGURE 6 - Specular reflections. (a) Bright vertical line. (b) Screen tilted $20^{\circ}$ reflects specular rays downwards. Other mirrors omitted for clarity. As the viewer is located at the virtual image of the projector, keystoning is not perceptible.

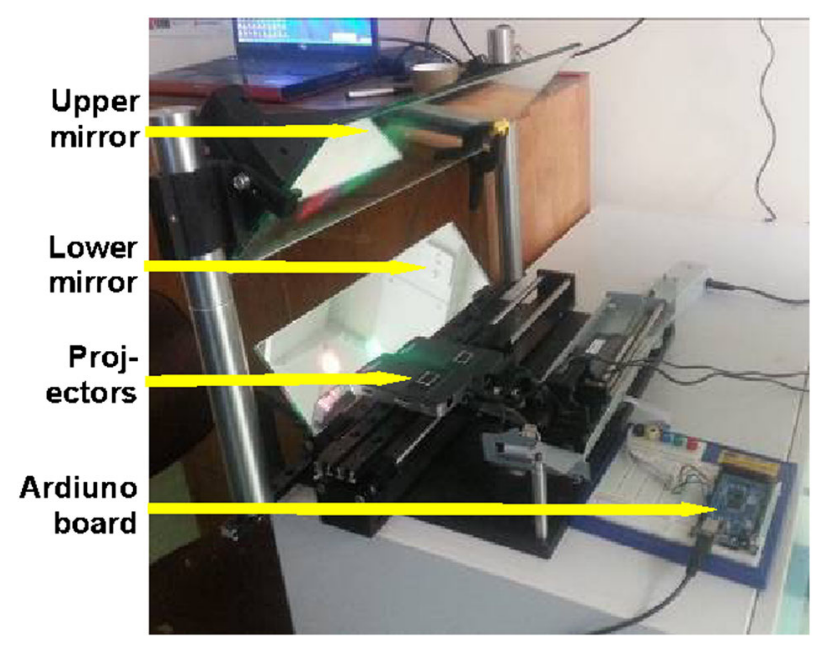

FIGURE 7 - Display mechanisms. This shows the two picoprojectors, the tracking mechanism, and the mirrors. The Arduino board uses the encoder in the printer mechanism to control a DC motor.

this is relatively small compared with the interpupillary distance (IPD). The stage consists of the mechanism taken out of a printer and thus allows $210 \mathrm{~mm}$ movement, which is the width of a sheet of A4 paper. The use of an inkjet printer cartridge mechanism is convenient as it includes a DC motor, belt drive, and incremental encoder with quadrature output for direction sensing. The display also includes a carriage with the projectors mounted on it and a mirror assembly.
As the viewer moves, the projectors and hence their projected images also move by the same amount if no correction is made. This effect is disconcerting to viewers as they become aware of the process of tracking because of the image moving relative to the frame of the screen. This can be overcome by two different methods: a linear or a pantograph mechanism.

\subsection{Linear mechanism}

The first method is to display a square image in the 16:9 aspect ratio frames from the picoprojectors and laterally shifting their positions in accordance with the head tracker output so that the left/right image pair always occupies the same position on the screen. The operation of this method is shown in Fig. 8(a), and the resulting stationary image on the screen for the two extreme head positions is demonstrated in Fig. 8 (b) where the image on the screen is adjacent to the tape marker in each case. Although the image is small in this case, it is possible to make the image fill the complete height of the frame if required.

\subsection{Pantograph mechanism}

The disadvantage of shifting the images within the projector frames is that the images have a reduced width due to the black regions that are required either side (Fig. 8(a)). If the image position is kept stationary by mechanical means, then the complete width of the frame can be utilized. This could be achieved with the use of a curved track for the projectors to follow, but this is reasonably difficult to construct.

A simpler method is to alter the directions of the projectors as they traverse the display. This can be achieved by mounting the projectors on a trapezoidal pantograph mechanism as shown in Fig. 9. In this case, the printer mechanism is coupled to the pantograph via a connecting rod, and it was found that it was simpler to control the motor if the standard DC motor was replaced by a stepper motor with the motor driver receiving its input from an Arduino board controlled by the head tracker.

The pantograph mechanism comprises two arms that have fixed pivots at positions A and B and a moving connecting link attached to moving pivots at $\mathrm{C}$ and $\mathrm{E}$. As the distance between $\mathrm{A}$ and $\mathrm{B}$ is greater than the distance between $\mathrm{C}$ and E. the connecting link on which the projectors are mounted rotates into position $\mathrm{C} \mathrm{E}$ at the full extent of the travel.

This enables the axis of the projectors to remain close to a fixed position as they move laterally. The rotating action of the link CE enables the perpendicular line from its center to remain close to a given point on a screen in front of it over a range of lateral movement.

Figure 10(a) shows the way in which the mechanism has a similar action to a convex lens where marginal rays focus closer to the lens because of spherical aberration. Increasing the distance between the fixed pivots from $A_{L} B_{L}$ to $A_{S} B_{S}$ decreases the "focal length" from $\mathrm{DF}_{\mathrm{L}}$ to $\mathrm{DF}_{\mathrm{S}}$. 

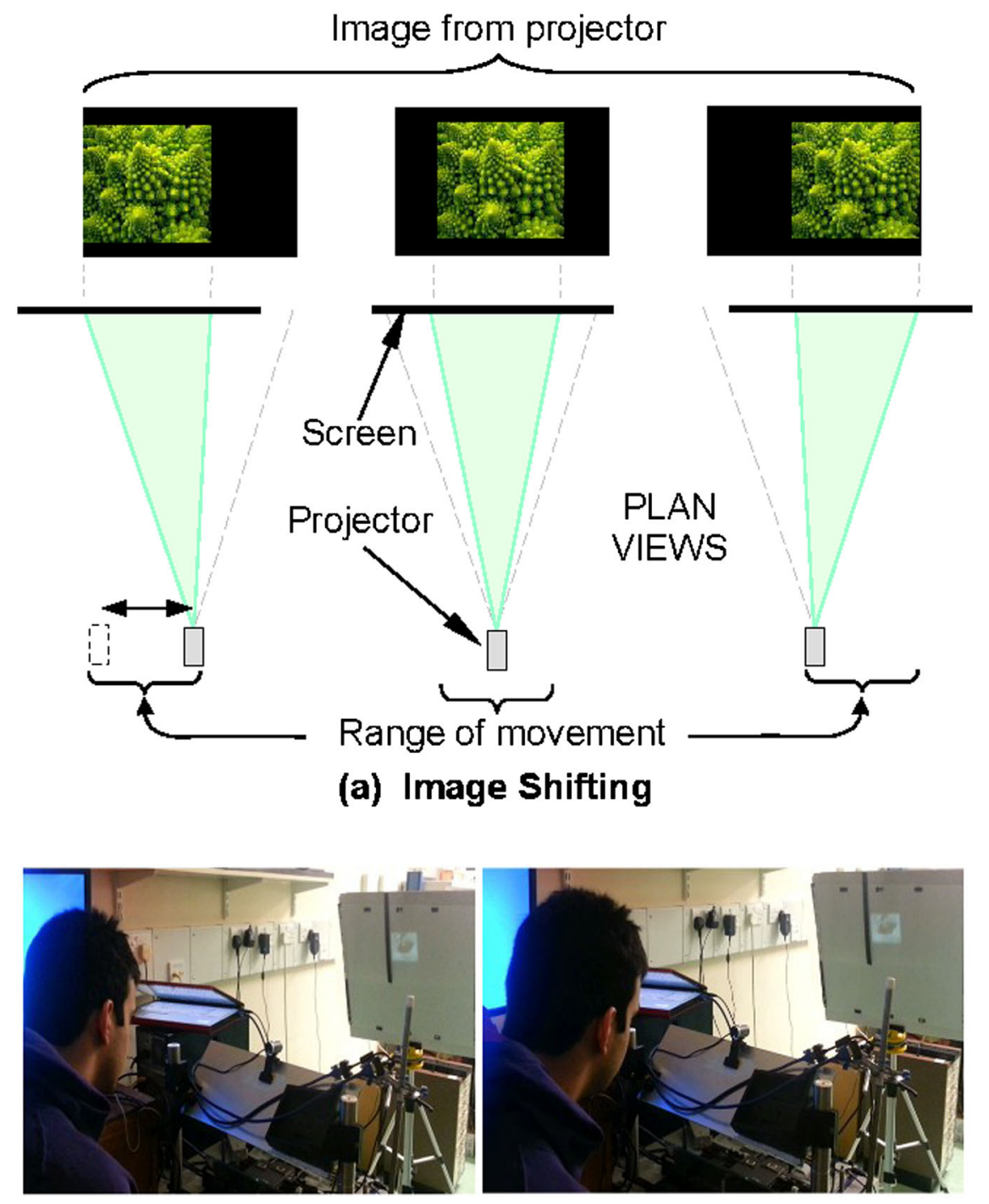

(b) Image Stabilisation in Action

FIGURE 8 - Image position stabilisation. (a) The image remains steady on the screen if software is used to shift its position in the frame in accordance with projector position. (b) Stabilization in action.

Figure 10(b) shows that for a viewer distance of $1.5 \mathrm{~m}$, the optimum distance between $\mathrm{A}$ and $\mathrm{B}$ is $322 \mathrm{~mm}$ for an arm length (AC and $\mathrm{BE}$ ) of $300 \mathrm{~mm}$. It can be seen that the lateral deviation is only $\pm 2 \mathrm{~mm}$. These measurements were made by mounting a laser at point $\mathrm{D}$ and noting the position of its beam on a screen $1.5 \mathrm{~m}$ away.

\section{$5 \quad$ Head tracker}

As the viewer is located close to a given distance from the screen, then the image of the viewer's head that is used to determine the positions of the eyes can be carried out with a single camera as we do not need to know the $\mathrm{Z}$ coordinates. The algorithm detecting the eyes looks for Haar-like features. ${ }^{29}$ It is assumed that during the procedure of face detection, the specific rectangular Haar-like features are constantly shifting and sliding in the detected image. Each time the face is detected, the characteristics of the region that are defined in the program used will be calculated and then use a specific mechanism to filter the particular characteristics.

The eye detection algorithm utilizes a cascade classifier working with the Haar-like features. Positive samples (with eye images) and negative samples (without eye images) are given to train the cascade classifier before detection. The typical distribution of eye pair region on a Haar-like feature set is recorded in cascade classifier parameters. During detection, the captured image that is transformed to gray-scale before searching is split into image blocks and detected for eyes in sequence. $^{30}$

A scan window determines the size of each image block. By putting the distribution of each image block on the face region and then subtracting the pixel sum in the black area from 


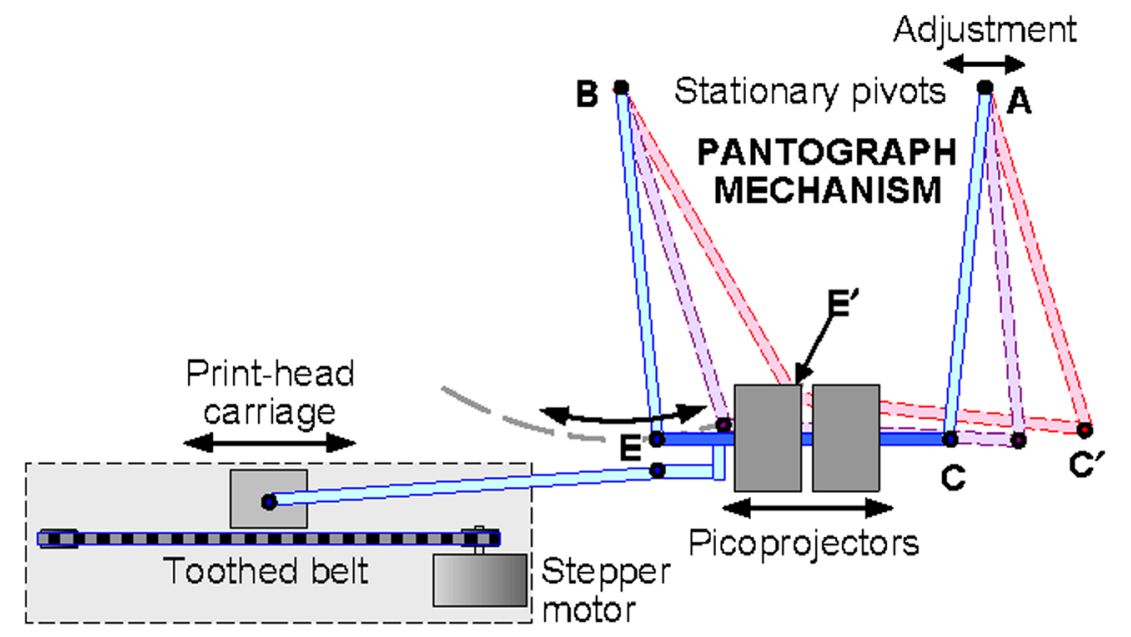

PRINTER MECHANISM

FIGURE 9 - Picoprojector mechanism. The linear motion of the printer mechanism is converted into curved motion by the pantograph. This enables the images to remain virtually stationary on the screen.

the pixel sum in the white area, the resulting value is the socalled face feature value. If we put the image block into a non-face area, then the calculated values should be different from the face feature values, and the more different the better. If most of the distribution is matched, the image block is considered to have successfully captured an eye pair region, and vice versa.

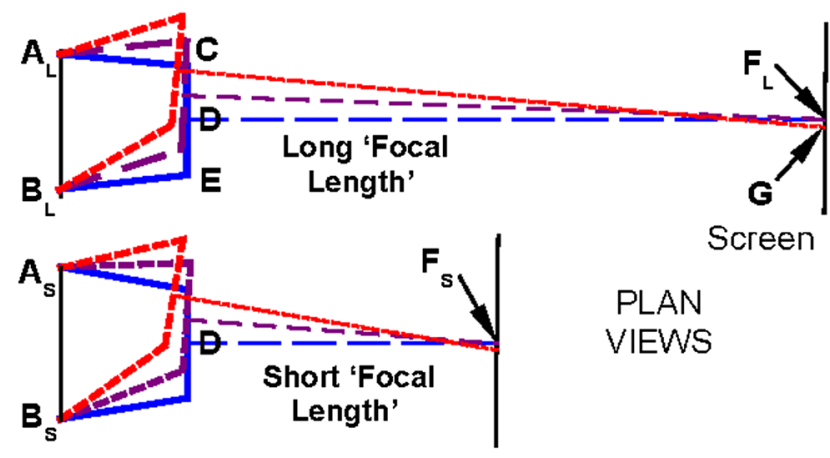

(a) Lens Equivalent

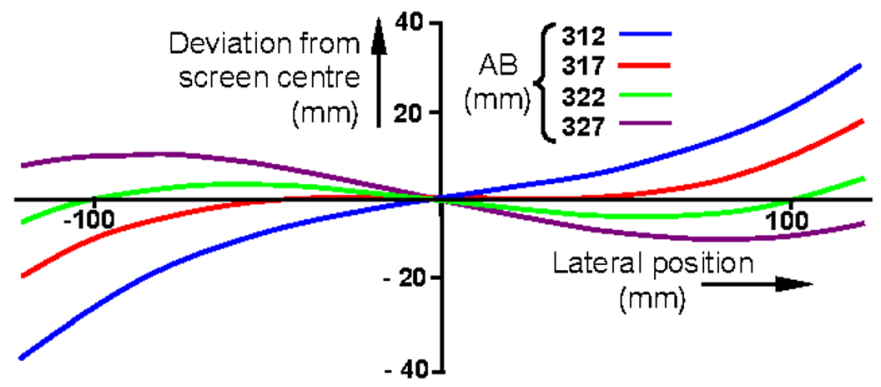

(b) Optimum Adjustment for $1.5 \mathrm{~m}$

FIGURE 10 - Pantograph. (a) It has similar performance to a convex lens, in terms of "focusing" and spherical aberration. (b) Optimum adjustment for $1.5 \mathrm{~m}$ "focal length" - in this case $A B=317 \mathrm{~mm}$ for $300 \mathrm{~mm}$ arm length.
In order to overcome problems caused by varying ambient lighting conditions, the image of the viewers' head is captured with an infrared camera with the head illuminated by infrared diodes located close to the viewer. The illumination comprises two modules, located either side of the head with each module having 15 LEDs that have a maximum current rating of $100 \mathrm{~mA}$.

The camera used is one that was originally a visible light camera where the visible band pass filter has been replaced with an infrared band pass filter with the spectral characteristic shown in Fig. 11. The spectral output of the LEDs is also shown on the figure, and it can be seen that the output is contained within the filter characteristic and that virtually all the visible spectrum is outside the characteristic.

By using an LED with a wavelength of $830 \mathrm{~nm}$, which is close to the visible range, the appearance of the image produces a monochromatic image that is very similar in

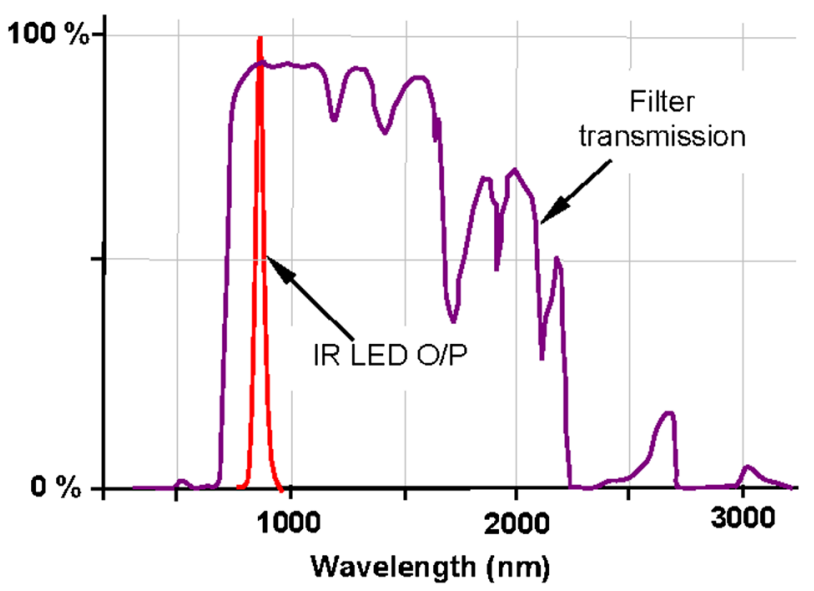

FIGURE 11 - Infrared filter and LED spectra. The LED output spectrum is contained within the filter transmission characteristic that has a sharp cutoff between the LED output and the longest visible wavelength. 
appearance to the visible light monochromatic image. This means that the same head tracking algorithms can be used.

The advantages of using this approach can be seen in Fig. 12. In Fig. 12(a), the infrared LEDs are switched off. It can be seen that, as expected, a dim image is only seen when daylight illuminates the viewer's head. In Fig. 12(b), it can be seen that the appearance of the head varies very little with very different ambient illumination levels.

As there are a large number of LEDs that are capable of passing a relatively high current, and these are located close to the viewer's eyes, the safety of this arrangement must be considered. Infrared can be particularly hazardous because the body's protective "blink reflex" response, and the involuntary control of the iris diameter is triggered only by visible light. Although infrared LEDs are not as dangerous as lasers, their invisibility might still cause trouble if not treated with care. It was noted that there is a warning given on the manufacturer's datasheet for the $3^{\circ}$ divergence angle version of $40^{\circ}$ LED of our display.

Sufficiently, powerful radiation in the visible and NIR range $(400-1400 \mathrm{~nm})$ will penetrate the eye and may cause heating of the retina. Radiation with wavelengths less than $400 \mathrm{~nm}$ and greater than $1400 \mathrm{~nm}$ is largely absorbed by the cornea and lens and can possibly cause the development of cataracts or burn injuries. ${ }^{31}$ As the potential damage is to the retina, that is, from images of the diode, then the consideration of the effect of a single LED and not the complete array is relevant as each diode will be focused separately in different region on the retina. In the worst-case situation where the eye is close to the LED, the emitting area of $0.3 \mathrm{~mm}$ square is resolved as a small image so that the power density on the retina is then unaffected by the distance of the LED. When the LED is very close to the eye, the defocusing of the retinal image reduces the danger. ${ }^{32}$ Safety of infrared LEDs is a fairly complex subject, but in order to keep a good margin of safety within the ICNIRP (International Commission of Non-Ionizing Radiation Protection) guidelines, ${ }^{33}$ the current through each diode is limited to $50 \mathrm{~mA}$; this corresponds to a radiant intensity of $27 \mathrm{Mw} / \mathrm{sr}$.

\section{Results}

As the screen comprises a retroreflector, it is likely that an image formed on it will be affected by reflections within it; also, the vertical diffuser that is located $10 \mathrm{~mm}$ in front of it will cause blurring in the vertical direction. It is expected that a certain degree of image degradation will occur and this was determined using a 1951 USAF Resolution Target. ${ }^{34}$ Figure 13(a) shows the projector image on a white screen with Lambertian distribution where the resolution in the photograph is dependent principally on the projector resolution and to a lesser extent on the resolution of the camera used to capture the image. Although the USAF resolution target is a relatively crude method of quantifying the performance

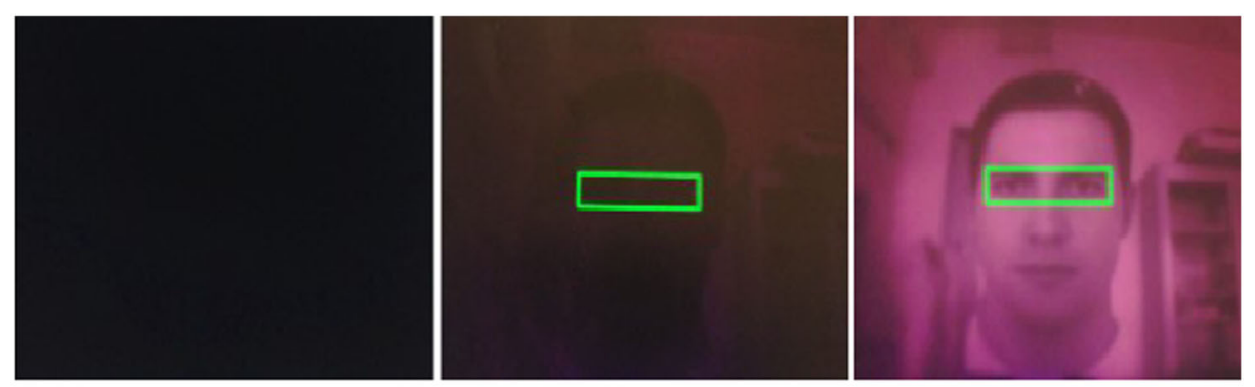

(a) Infrared illumination off
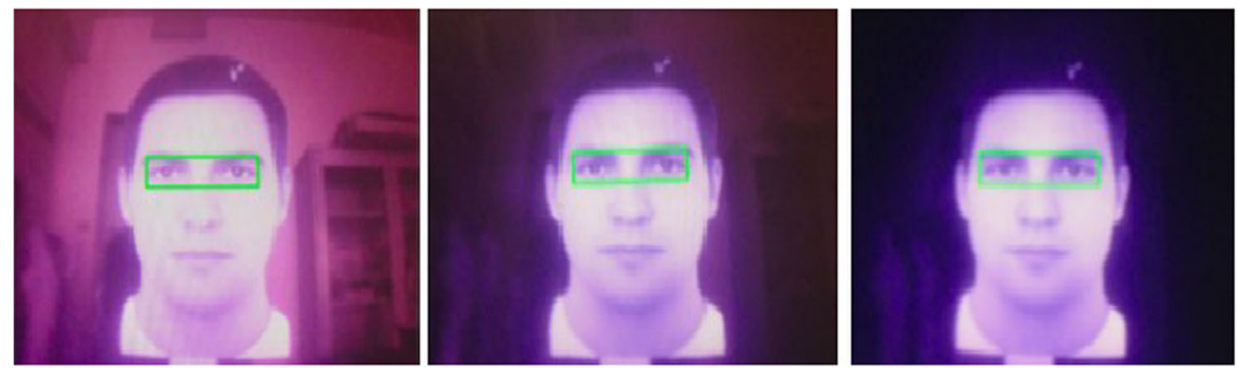

(b) Infrared illumination on

\section{I - No illumination}

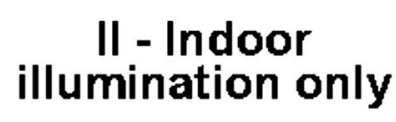

FIGURE 12 - Infrared illumination. (a) With ambient illumination, only the head is not reliably tracked. (b) With infrared illumination, the image of the head remains steady over a range of lighting conditions. 


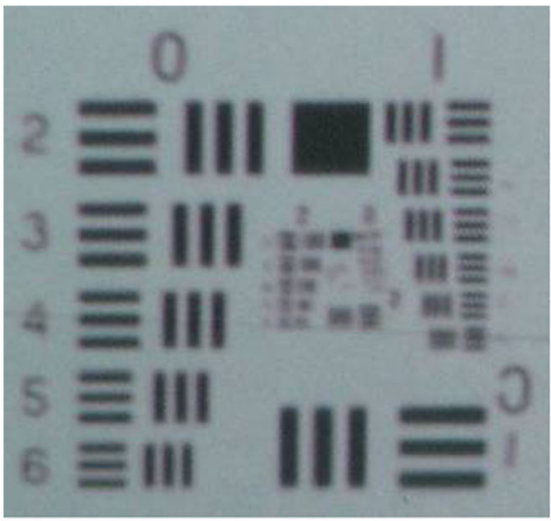

(a) White Screen

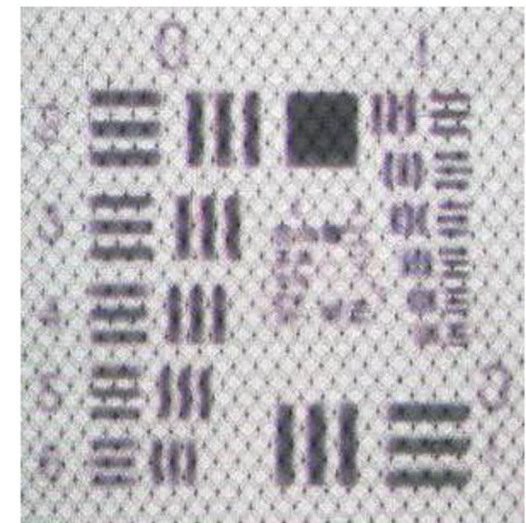

(b) Retroreflector Screen

FIGURE 13 - Resolution target. (a) Projected on to white screen. (b) Projected on to retroreflector screen, slightly worse resolution in the bars on the right, a visible structure, and a high-resolution loss on the numbers on the left.

of the display and there is no absolute scale in the figure, the relative degradation can be clearly determined visually. In Fig. 13(b) the additional effect of screen artifacts can be seen. The horizontal and vertical bars on the left of the numbers on the right are barely legible. The diamond pattern is part of the structure of the retroreflector back surface that has a pitch of $4 \mathrm{~mm}$ and is clearly visible at the $1.5 \mathrm{~m}$ viewing distance.

Crosstalk was measured by showing a white image on the left channel with the right projector switched off and traversing the exit pupil with a power meter. The power meter detector has a $10 \mathrm{~mm}$ diameter capture area and was mounted on a motorized stage. The procedure was repeated with a white image on the right channel and the left projector off. The normalized plots are shown in Fig. 14. The left channel crosstalk $=\mathrm{BC} / \mathrm{AC}$ and right channel crosstalk $=\mathrm{EF} / \mathrm{DF}$. The values for the left and right channel are $8.0 \%$ and $5.8 \%$, respectively. The appearance of the screen with " $L$ " and "R" test images is shown in Fig. 15 where a slight ghost image is seen in the left image. The vertical lines in Fig. 14 on which the measurements are taken represent points that are $65 \mathrm{~mm}$ apart, which is close to the average IPD and is the

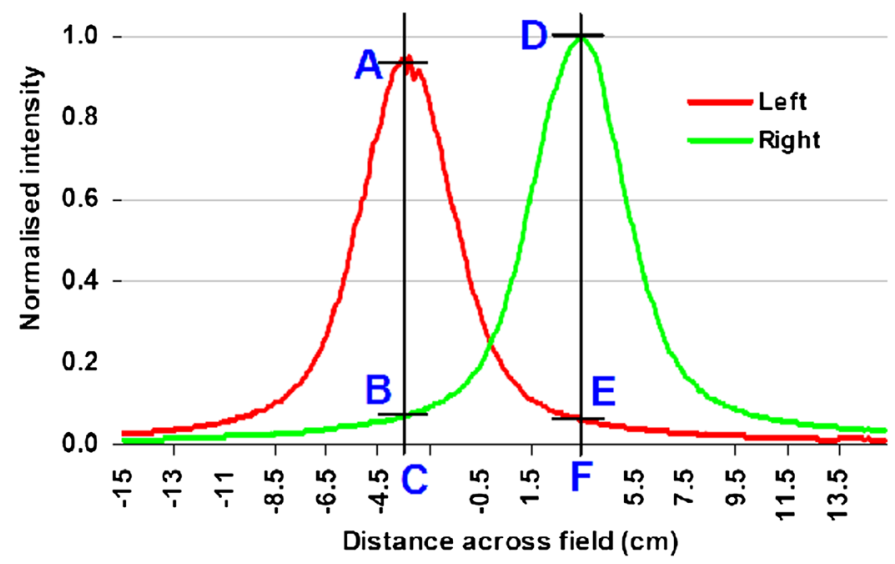

FIGURE 14 - Crosstalk. Intensity profiles of the left and right exit pupils at $1.5 \mathrm{~m}$ from the screen. The values for the left and right channels are $8.0 \%$ and $5.8 \%$, respectively. separation of the peak outputs of the left and right exit pupils. The head tracker ensures that these peaks follow the positions of the user's eyes. As the projectors are mounted with a lateral separation of $65 \mathrm{~mm}$, which is around the average adult eye separation, the projectors are toed-in so that the left and right images overlap on the screen. This causes keystone distortion where the height of the image at the edge furthest away from the projector lens is greater than the height at the nearest edge (Fig. 16).

A very approximate rule of thumb regarding stereoscopic image capture with a camera pair is that the separation between the cameras does not exceed 1/30 of the distance of the subject. ${ }^{35}$ The rule applying to capture will also apply to projection as the same level of distortion is introduced because of similar geometry. This means that the rule of thumb can be used as a guide to give an indication of the level of

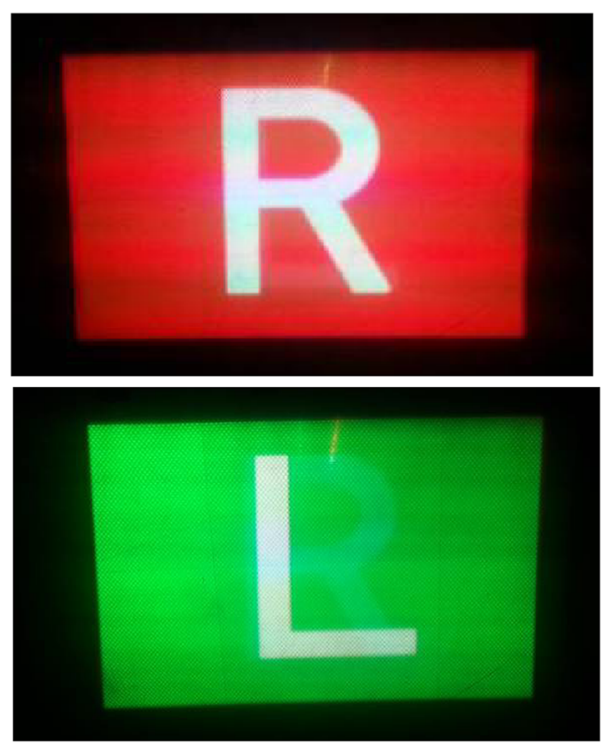

FIGURE 15 - Images on screen. Taken at a camera separation of $65 \mathrm{~mm}$, the approximate average eye spacing distance. Some ghosting is visible in the left channel. 


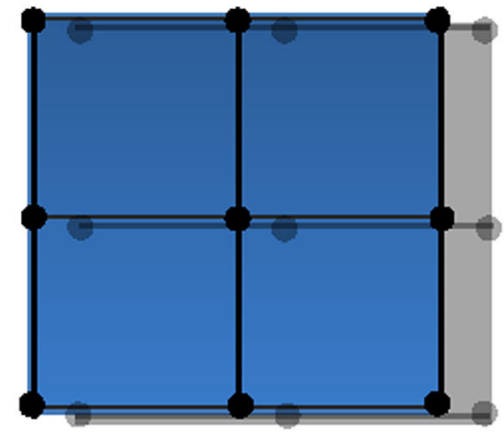

\section{(a) Ideal image on} screen

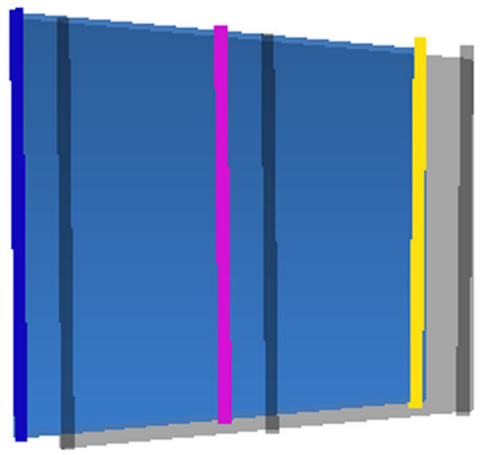

Projector to right

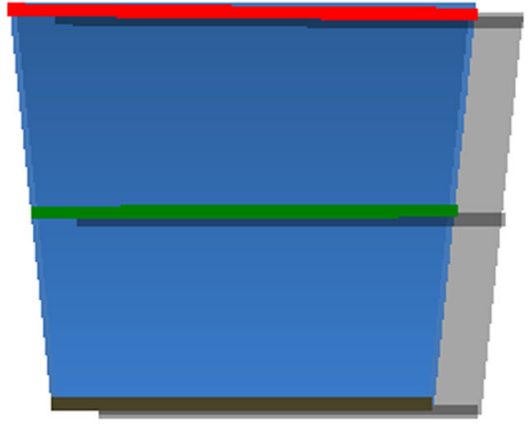

(b) Horizontal keystoning

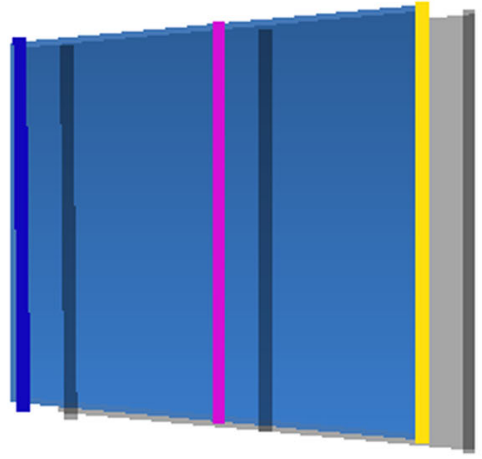

Projector to left

\section{(c) Vertical keystoning for left eye and right projectors}

FIGURE 16 - Keystoning. (a) Image from projector on axis, (b) image from projector below axis, and (c) images from projectors to the right and to the left of the axis.

tolerable keystone distortion. In the prototype, the projector separation is $65 \mathrm{~mm}$, and the screen distance is $1.5 \mathrm{~m}$. This is equivalent to a distance to camera separation of $23: 1$, which is of the same order as the rule of thumb. Although there is considerable literature on horizontal disparity, for example, Cormack and Fox ${ }^{36}$ and Williams and Parrish ${ }^{37}$, it is not a particular issue with this display, however vertical disparity caused by keystone distortion is a potential problem. Excessive vertical disparity affects the ability of the brain to fuse the images. ${ }^{38}$ Keystone distortion is a significant issue if it differs between the two eyes and has been investigated for displays similar to the authors ${ }^{39}$, and measures for correcting it have been studied. ${ }^{40}$

The resulting images are shown in Fig. 17, where the keystoning shows as different colored regions at the top and bottom of the image. The images used in this case were a white letter "R" on a red background with a black graticule superimposed for the right channel and a white letter " $L$ " on a green background with a black graticule superimposed for the left. The projectors are set up by adjusting them so that there are no red or green fringes visible on the graticules.

Informal user trials were carried out on eight subjects where a series of 10 still images was presented to each of

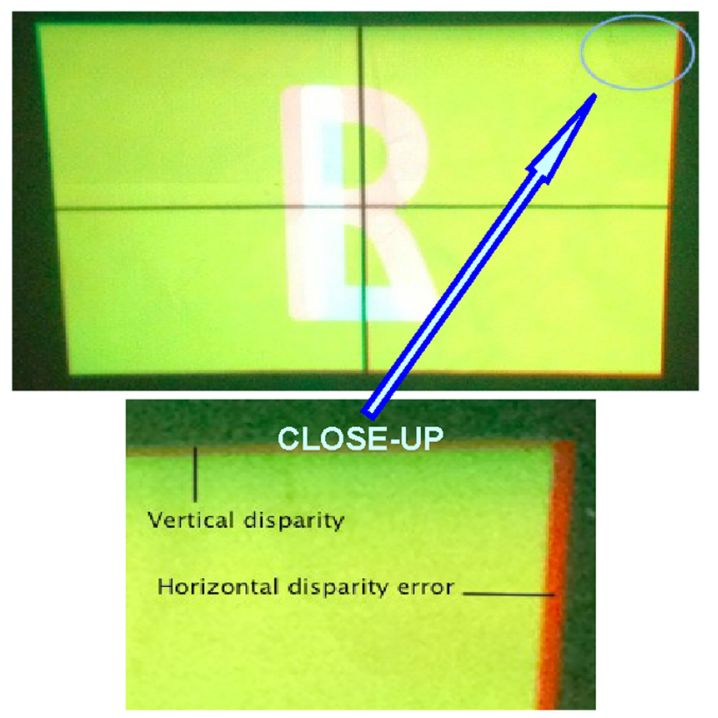

FIGURE 17 - Disparities. The vertical and horizontal disparities at the screen edges are indicated by the red (or green) fringes. Correct alignment is indicated by a black (not red or green) graticule.

them. They were not given a stereo acuity test, but the ease with which stereo could be perceived for the majority of the 
images, at least according to their response to the question "can you see 3D?," implied that none of them were stereo blind. A comparison between monoscopic and stereoscopic images was made by presenting the same images to both eyes and then presenting stereo pairs. Out of the total of 80 replies, $88 \%$ were positive in stating that the subjects preferred the experience of viewing the images stereoscopically.

Although the sample number was small, and comparison was made between monoscopic and stereoscopic images on the same display system, the results indicate that significant improvement has been achieved with the addition of stereo.

One explanation for vertical disparity not being a problem in the trials could be that this disparity is at its maximum at the corners, whereas the region of interest where viewers fixate is generally located near the center of the image.

It is unlikely that IPD variation will be an issue as a study ${ }^{41}$ of anthropometric data on almost 4000 subjects gives a mean IPD of $63.36 \mathrm{~mm}$ with absolute minimum and maximum values of 52 and $78 \mathrm{~mm}$ and a standard deviation of $3.832 \mathrm{~mm}$.

The close-up in Fig. 17 shows that the horizontal disparity error is considerably greater than the vertical disparity error; however, this only produces depth distortion effects, which have a lesser effect on perceived image quality.

\section{$7 \quad$ Future applications}

For single user autostereoscopic display applications, this approach possibly might not provide the most practical solution. However, it could have uses as a multi-user display where there is a requirement to provide a unique image-pair to each user, for example, to provide motion parallax or for security purposes. A separate projector-pair would be required for each user, but as these are relatively inexpensive, cost is unlikely to be an issue.

Head tracking from a single tracker would be difficult given the distance from the screen to the users which would be in the region of several meters. As there are projectors for each user, there could be local single-target trackers located in the same housings as these projectors. A possible set-up for nine users is shown in Fig. 18. As the screen size is considerably larger than the prototype screen, picoprojectors are unlikely be sufficiently bright, and larger projectors would be required. However, the effective gain of the retroflecting screen lowers the output requirement considerably compared with that for a conventional screen.

If a large screen is used as shown in the figure and the screen width is large in relation to its distance, then the exit pupils will not have the depth of those of the prototype. Limited viewer movement in the $\mathrm{Z}$ direction could be overcome with the use of $\mathrm{Z}$ tracking and moving the projectors in both the $\mathrm{X}$ and $\mathrm{Z}$ directions. The projectors would have to move over an area that is the same size as the viewer movement, but this will be limited as the viewers' head positions are constrained by the fixed seat positions.

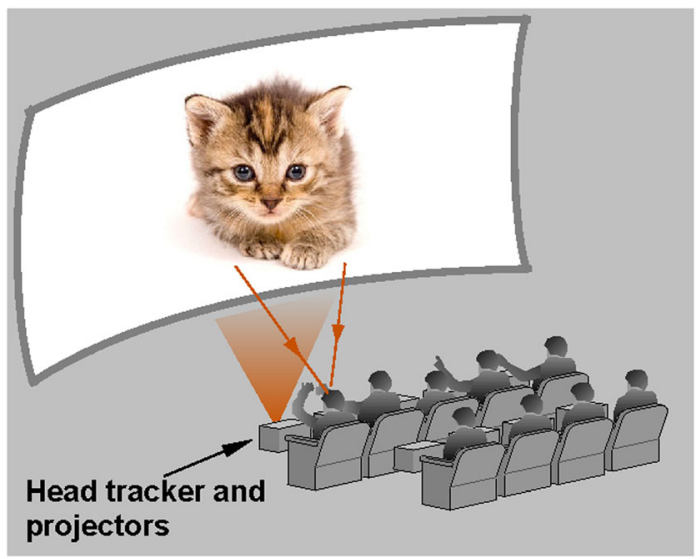

FIGURE 18 - Possible multiple user configuration. A separate head tracker/projector-pair unit for each viewer could enable each one to see a unique stereo pair.

Another potential challenge with this approach is that the return beams from the screen must be highly collimated in order to keep crosstalk within acceptable limits. With typical viewing distances of several meters, the angular spread of the reflected beam must be small in order to prevent too much image leakage to the adjacent eye.

Another future direction could be to incorporate $Z$-axis movement/tracking, head-rotation tracking, and potentially vertical tracking so that motion parallax cues can be as accurate and robust as possible. This could then allow for high-precision virtual reality tasks such as robotic surgery, bomb disposal, and so on without the cumbersome eyewear, and yet that satisfies as many perceptual cues to depth as possible using stereo technology.

\section{Conclusions}

The proposal of the use of a retroreflector screen in a 3D display application is not new, ${ }^{1,42}$ but the use of picoprojectors in conjunction with head tracking where the image position is stabilized using the head position information has not been previously reported. An inexpensive autostereoscopic display is produced that provides a bright image by exploiting the geometrical properties and the inherent gain of a retroreflecting screen. The cost of the remainder of the prototype display is also low as it can use the standard mechanism from a printer.

Head tracking is enabled with the use of a single inexpensive webcam. The principal disadvantage of the current display is the appearance of the diamond pattern on the screen. The use of a more suitable material will be further investigated. Apart from the patterning, the current material has ideal optical properties for this application. There are bead-type retroreflectors available, but their performance in terms of reflectance and reflected beam collimation generally tends to be inferior. 
The work constitutes the initial stage of a research project to establish the viability of this approach through the objective measurement of image position stabilization and of crosstalk between the image pair and the subjective evaluation in the form of informal user trials with a small number of subjects. The results are sufficiently positive to justify the continuation of this line of research.

\section{Acknowledgments}

The authors wish to thank Klaus Hopf of Fraunhofer HHI for his invaluable help with setting up the head tracker.

\section{References}

$1 \mathrm{~T}$. Baloch. "Method and apparatus for displaying three-dimensional images," United States Patent No. 6,201,565 B1 (2001). http://www. holografika.com/ (accessed 28 September 2014).

2 C. Moller and A. R. L. Travis. "Flat panel time multiplexed autostereoscopic display using an optical wedge waveguide." Proceedings of the 11th International Display Workshops, Niigata, Japan 1443-1446 (2004).

3 D. Lanman et al., "Content-adaptive parallax barriers: optimizing duallayer 3D displays using low-rank light field factorization," MIT Media Lab - Camera Culture Group. http://cameraculture.media.mit.edu/hr3d/ content-adaptive.pdf (accessed 28 September 2014).

$4 \mathrm{~W}$. A. IJsselsteijn et al., "Human factors of 3D displays," 3D Videocommun: Algorithms, Concepts, and Real-Time Systems in HumanCentred Commun John Wiley, ISBN 0-470-02271-X, 219-234 (2005). DOI: 10.1002/0470022736.ch12.

5 C. Van Berkel et al., "Multiview 3D-LCD," "SPIE Proceedings, Stereoscopic Displays and Virtual Reality Syst IV" 2653, 32-39 (1996), DOI: 10.1117/12.237437.

6 W. IJzerman et al., "Design of 2D/3D switchable displays," in Proc of the SID 36, No. 1, 98-101, May (2005), DOI: 10.1889/1.2036616.

7 N. A. Dodgson, "Multi-view autostereoscopic 3D display," Stanford Workshop on 3D Imaging 27 January (2011), DOI: 10.1007/978-1-44199964-1_13 http://www.cl.cam.ac.uk/ nad10/pubs/Stanford3D-2011.pdf (accessed 28 September 2014).

8 D. M. Hoffman et al., "Vergence-accommodation conflicts hinder visual performance and cause visual fatigue," J. Vis. 8, No. 3, (2008). DOI: 10.1167/8.3.33.

9 G. Jones et al., "Controlling perceived depth in stereoscopic images," Proceedings of SPIE 4297, 42-53 (2001). DOI: 10.1117/12.430855.

10 D. Kim et al., "Depth adjustment for stereoscopic images subjective preference evaluation," J. Electron. Imaging. 20, No. 3, 033011 August 19, (2011). DOI: 10.1117/1.3624499.

11 A. Schwartz, "Head tracking stereoscopic display." Electron Devices, IEEE Transactions, Proc IEEE Intern Disp Res Conf. 33, No. 8, 141144 (1985). DOI: 10.1109/T-ED.1986.22628.

12 G. Woodgate et al., "Observer tracking autostereoscopic 3D display systems." SPIE Proceedings, "Stereoscopic Displays and Virtual Reality Syst IV" 3012, 187-198 (1997). DOI: 10.1007/978-0-387-79335-1_7.

$13 \mathrm{~S}$. A. Benton et al., "Micropolarizer-based multiple-viewer autostereoscopic display." SPIE Proc 'Stereoscopic Displays and Appl X' 3639, 76-83, (1999). DOI: 10.1145/344779.344933.

14 Microsoft (2010). "The wedge - seeing smart displays through a new lens" http://www.microsoft.com/appliedsciences/content/projects/wedge.aspx (accessed 28 September 2014).

15 "LG introduces the world first Glasses-Free 3D monitor with eye-tracking technology," http://en.akihabaranews.com/101207/displays/g-introducesthe-world-first-glasses-free-3d-monitor-with-eye-tracking-technology (accessed 28 September 2014).

16 "Review of the Toshiba Qosmio F750 autostereoscopic 3D laptop," http:// 3dvision-blog.com/6505-review-of-the-toshiba-qosmio-f750-autostereoscopic-3d-laptop/ (accessed 28 September 2014).

17 R. Fisher. (1996). US Patent No. 5,572,229. Washington, DC: U.S. Patent and Trademark Office.
18 R. Kijima and T. Ojika. "Transition between virtual environment and workstation environment with projective head-mounted display." Proceedings of IEEE 1997 Virtual Reality Annual International Symposium. CA, Los Alamitos (1997), 130-137.

19 J.P. Rolland et al., "Development of head-mounted projection displays for distributed, collaborative augmented reality applications," Presence: SI Immersive Projection Technol 14, No. (5), 528-549 (2005).

20 R. Martins et al., "A mobile head-worn projection display," Opt. Express 15, 14530-14538 (2007).

21 M. Cobb Joshua et al., "High-resolution autostereoscopic immersive imaging display using a monocentric optical system," Proc. SPIE 5006, Stereoscopic Displays and Virtual Reality Syst X 92, May 29, (2003). DOI: 10.1117/12.474123.

22 T. Okoshi. "Three dimensional imaging techniques," New York: Academic Press, 1976, pp 127-131.

23 K. Peck and M. D. Morris. "Optical properties of RRA in stereoscopic applications," Rev. Sci. Instrum 58, No. 2, February (1987).

$24 \mathrm{R}$. Zhang and H. Hua, "Imaging quality of a retro-reflective screen in head-mounted projection displays," J. Opt. Soc. Am. A, Vol. 26, 05 May (2009).

25 R.B. Nilsen and M. Hanrahan, "Retro-reflective articles with multiple size prisms in multiple size locations," United States Patent 6,139,158, 31 October, 2000.

26 "Reflective glass beads," Virginia Department of Transport. http://www virginiadot.org/business/resources/materials/mcs_study_guides/bu-matpavemarkch2.pdf (accessed 28 September 2014).

27 A. J. Woods, "Crosstalk in stereoscopic displays: a review," J. Electron. Imaging 21, No. 4, (2012). DOI: 10.1117/1.JEI.21.4.040902.

28 P.J.H. Seuntiens et al., "Perceptual attributes of crosstalk in 3D images," J Imaging Sci and Tech 53, No. 3, (2009). DOI: 10.1016/j.displa.2005. 06.005 .

29 Chen Duan-Sheng and Liu Zheng-Kai, "Generalized haar-like features for fast face detection," 6th Int Conf Mach Learn Cybern 4, 2131-2135 (2007). DOI: 10.1109/ICMLC.2007.4370496.

30 Intel, Integrated Performance Primitives 7.1 Reference Manual. available at: http://software.intel.com/sites/products/documentation/doclib/stdxe/ 2013/composerxe/ipp/ipp_manual/IPPI/ippi_ch14/ch14_haar_features. htm\#fig. 14-13 (accessed 28 September 2014).

$31 \mathrm{~W}$. T. Ham et al., "Retinal sensitivity to damage from short wavelength light," Nature 260, 153-155 (1976).

32 N. Kourkoumelis and M. Tzaphlidou, "Eye safety related to near infrared radiation exposure to biometric devices," Scientific World Journal 11, 520-528 (2011) Mar 1. DOI: 10.1100/tsw.2011.52

33 D. H. Sliney, "ICNIRP statement on light-emitting diodes (LEDs) and laser diodes: implications for hazard assessment," Health Phys. 78, 744-752 (2000).

34 http://www.edmundoptics.com/technical-support/testing-targets/choosing-the-correct-test-target/?\&pagenum $=4-. \quad$ Edmond Optics (Test Targets), (accessed 27 November 2013).

35 T. Dashwood, "A beginner's guide to shooting stereoscopic 3D," September (2011). http://www.dashwood3d.com/blog/beginners-guide-to-shooting-stereoscopic-3d/ (accessed 15 August 2013).

36 R. Cormack and R. Fox, "The computation of retinal disparity and depth in stereograms," Percept. Psychophysics 38, 375-380 (1985).

37 S. P. Williams and R. V. Parrish, "New computational control techniques and increased understanding for stereo 3-D displays," Proc SPIE 1256 73-82 (1990)

38 A. J. Woods et al., "Image distortions in stereoscopic video systems," SPIE Proc. Stereoscopic Displays and Appl IV 1915, September 23 (1993). doi:10.1117/12.157041.

39 E. W. Jin and M. E. Miller, "Tolerance of misalignment in stereoscopic systems," Proceedings of the International Congress of Imaging Science (ICIS)'06, Rochester, NY: Society for Imaging Science and Technology, pp. $370-373$ (2006).

40 Bauer A. et al., "Computational optical distortion correction using a radial basis function-based mapping method," Opt. Express 20, No. 14, 14906-14920 (2012).

41 N. A. Dodgson, "Variation and extrema of human interpupillary distance," Stereoscopic Displays and Virtual Reality Syst XI 5291, 36-46, May (2004).

$42 \mathrm{P}$. Harman, "Retroreflective screens and their application to autostereoscopic displays," SPIE Proc 3012, 0277-786X/971\$10.00 145, (1997). DOI: $10.1117 / 12.274451$. 


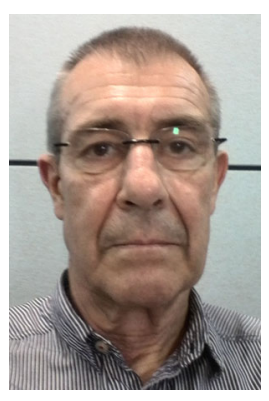

Dr. Phil Surman is a Visiting Senior Research Fellow in the Imaging and Displays Research Group at De Montfort University in the UK and a Visiting Research Associate at University College London. He received his $\mathrm{PhD}$ in the subject "Head Tracking Two-image 3D Television Displays" from De Montfort University in 2002 He has carried out research into glasses-free television displays for many years and holds the patents on the multi-user autostereoscopic television displays developed in the European Union-funded ATTEST, MUTED, and HELIUM3D projects.

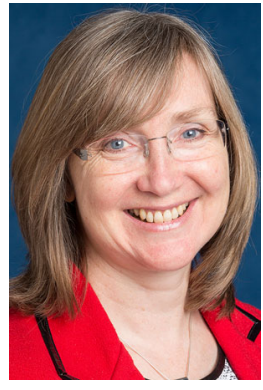

Sally Day studied for her DPhil at the Clarendon Laboratory, Oxford University. In 1992, she was awarded a Royal Society University Research Fellowship to work at $\mathrm{UCL}$, where she is now Senior Lecturer. She has around 80 refereed journal and conference publications. At UCL, in the Optical Devices and Systems laboratory in the Photonics group, her research has included liquid crystals in tunable microlenses, wavelength selective Fabry Perot filters, application of modeling software, design of Liquid Crystal on Silicon devices, cholesteric polarizers, large area displays for architectural applications, the use of liquid crystals in microwave devices, and the optical design of an autostereoscopic display. She is currently the UK Director for the Society for Information Display.

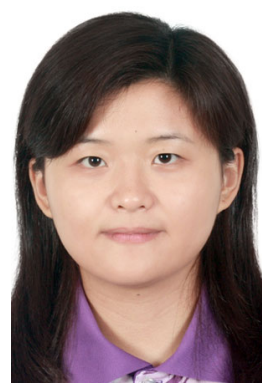

Xianzi Liu received her bachelor's degree in E-commerce Engineering with Law (joint program with dual degree) with a first class B.Eng. from Queen Mary, University of London and a B.Mgmt. from Beijing University of Posts and Telecommunications. She received her MSc in Internet Engineering from University College London in 2013.

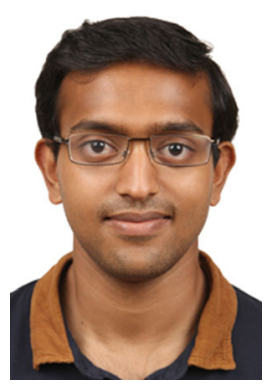

Joshua Benjamin received his M.Eng. in Electronic Engineering and Nanotechnology from University College London in 2013. He is currently pursuing an MRes in Photonic Systems Development at the UCL-Cambridge Centre for Doctoral Training (CDT); this is a scheme where the University of Cambridge and UCL have joined forces to develop the next generation of photonic systems, pioneering research with light.

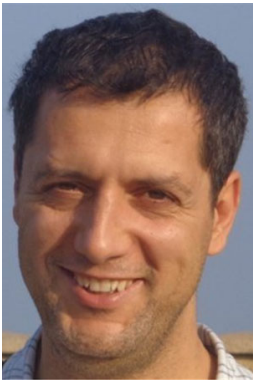

Hakan Urey is a full Professor at Koç University, Istanbul, Turkey, and a visiting Professor at Boğaziçi University and at Cambridge University during 2013. He received the BS degree from Middle East Technical University in 1992 and MS and PhD degrees from Georgia Institute of Technology in 1996 and in 1997. After completing his $\mathrm{PhD}$, he joined Microvision Inc. as Research Engineer and played a key role in the development of the Retinal Scanning Display technology. He was the Principal System Engineer when he left Microvision to join the faculty of engineering at Koç University in 2001. He was promoted to Associate Professor in 2007 and Professor in 2010

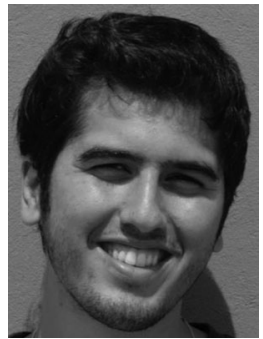

Kaan Aksit received a BS degree in Electrical Engineering from Istanbul Technical University in 2007 and an MSc in Electrical Power Engineering from RWTH Aachen University in 2010. He was a key member of the ITU solar car team during 2006 and 2007 where they won several trophies including best design. In 2009, he joined visual experiences group at the research center of Philips, Eindhoven, where his research interests included linear actuators, smell-o-vision devices, medical optics instrumentation, and optical sensing and sensors. In 2010, he is currently studying for his $\mathrm{PhD}$ in the Optics and Micro-Electro-Mechanical Systems group at Koç University where under Prof. Urey's guidance, he worked on the EU HELIUM3D project. 